\title{
Controlling the Movement of a Human Forearm based on Finite Time Optimal Regulator and Nonlinear Control System
}

\author{
Gamal A. Elnashar \\ Automatic Control Center \\ School of Engineering-Egyptian Armed Forces \\ 15 Yassien Raghb St., Nasr City, Cairo, Egypt
}

\begin{abstract}
Optimal human forearm trajectories structure was investigated using Optimal Regulator. First, the continuous dynamics of the human forearm were established on the basis of the relation between muscle torque and neural control signal and employed Riccati differential equation to obtain the optimal movement trajectory of the human forearm. The model and control of a human forearm is analyzed. In the advanced control of robotic manipulators, it is important for manipulators to track trajectories in a wide range of work place. If speed and accuracy is required, the control using conventional methods is difficult to realize because of the high nonlinearity of the robot system. In this work; we study the problem of human hand control carrying a mass. The equation of motion and the natural frequency of the forearm for small angular displacement are derived. We develop new methods that use vector fields in the controller construction for a set of nonlinear dynamical systems. The paper deals with compensate of non-linear system which has a similar idea as the method mentioned in linear system. We utilize an optimal regulator and suggest human arm activities under dynamic environments. Humans must pay compensation for loads arising from interaction with the physical environment. We have managed to design a control law for the non-linear arm-mass-system, in such a way that the representation of a closed loop system is affine, controllable, and a closed loop system is asymptotically stable. Throughout any motion, the forearm can be considered a one-link robot manipulator which could be exploited to benefit people with disabilities (missing extremities). An optimal controller calculates the motor command, which minimize the cost function for nonlinear arm dynamics. Therefore, the purpose of our study is to construct a computational model of arm movement. For this purpose, we use an optimal regulator as the model and compare simulated arm movements with actual nonlinear model human movements. In this paper, we present two approaches to human arm modeling: finite time optimal regulator control approach and modern nonlinear control approach. The latter one is formalized using Lie-Derivative based controllers.
\end{abstract}

\section{Keywords}

Two point boundary value problem, optimal regulator, Forearm Modeling, Nonlinear Controller, Vector Field, Lie derivatives

\section{INTRODUCTION}

The problems connected with the design of artificial arm replacements are far more difficult than those associated with the design of robotic arms or terminal devices. The design of artificial arms is a multidisciplinary effort. The design side needs knowledge of the mechanics of mechanisms, such as gears, levers, and points of mechanical advantage, and electromechanical design, such as switches, dc motors, and electronics [1]. When the someone wants to move the arm, the brain sends signals that first bond the chest muscles, which send an electrical signal to the prosthetic arm, instructing it to reposition. The procedure requires no more aware effort than it would for a someone who has an ordinary arm. In general, a person with a prosthetic arm can make only a few motions, often so slowly that many people use the arms only for limited activities. There is a separate motor for each movement. By far the most common actuator for electrically powered prostheses is the permanent magnet dc electric motor with some form of transmission [2]. In proportional control, the amount/intensity of a controlled output variable is directly related (proportional) to the amount of the input signal. For example, the output speed of a DC motor is proportional to the amount of voltage applied to its terminals. This is why DC motors are said to be speed controlled. This is also the reason why most of today's commercially available prosthetic components are speed controlled [3]. Output speed is comparative to the amount of input signal. Proportional control is used where a graded response to a graded input is required. In position control the position of the prosthetic joint is proportional to the input amount/intensity. The input amount/intensity might be the position of another physiological joint or a force level. If the position of another joint is used as the input then the system is known as a position actuated, position servomechanism. If the amount of force applied by some body part is the input, then the system is a force actuated, position servomechanism [4]. 

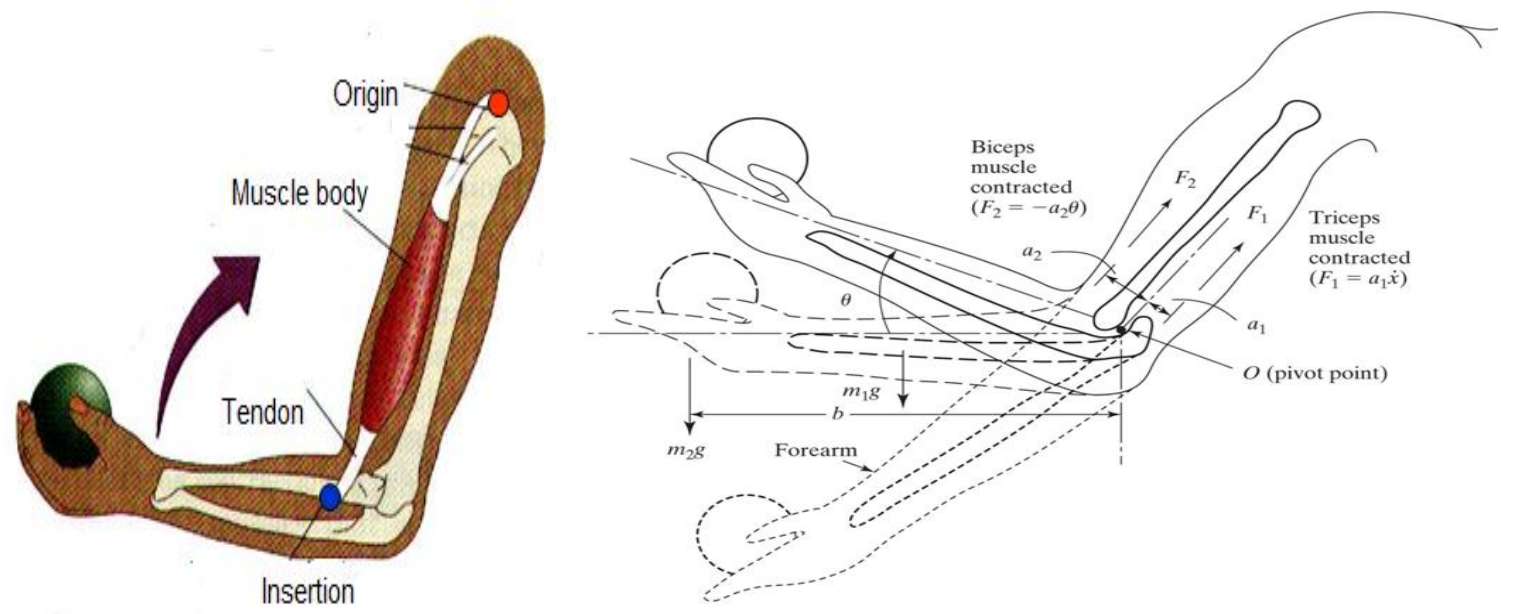

Fig 1: Forearm of a human hand carrying a mass.

With position control the amputee's ability to perceive and control prosthesis position is directly determined by his or her ability to perceive and control the input signal. A major disadvantage of position control is that, unlike velocity control, it must maintain an input signal to hold an output level other than zero. This means that power must be continuously supplied to the component to maintain a commanded position other than zero. This is one of the reasons why speed or velocity control is the main mode of control in externally-powered prosthetics today, despite the fact that it has been shown that position control for positioning of the terminal device in space is superior to velocity control. In control theory, a state observer is a system that provides an estimate of the internal state of a given real system, from measurements of the input and output of the real system[5-6]. It is normally computer-implemented, and provides the basis of many practical applications. Knowing the system state is necessary to solve many control theory problems; for example, stabilizing a system using state feedback. In most practical cases, the physical state of the system cannot be determined by direct observation. As a substitute, indirect effects of the internal state are observed by way of the system outputs. If a system is recognizable, it is possible to fully rebuild the system state from its output measurements using the state observer. In this day of digital circuits and microprocessor based controllers pulse width modulation is the preferred method of supplying a graded (proportional) control signal to a component. A PWM stream only requires a single digital output line and a counter on the microprocessor to be implemented, whereas a predictable analog signal (linear DC voltage level) requires a full digital-to-analog (D/A) converter. PWM techniques are used extensively in switchedmode power supply design and audio amplifiers and as such, there is a large array of resources available to the designer to choose from [8]. General properties of optimal solutions for the time-optimal control problem for nonlinear systems that are affine functions of the control(s) will be developed in which provides an introduction to some of the Lie derivativebased techniques that form the basis for geometric methods in optimal control. In this study, a model for a forearm performing a motion is presented, using a new controller technique based on vector fields. Furthermore, we evaluated three position controllers. The forearm bar of mass $m_{1}$ and length $b$ is shown in Figure 1. A mass $m_{2}$ is carried by the angular of the forearm of a human hand. During motion, the forearm can be considered to rotate about the joint (pivot point $\mathrm{O}$ ) with muscle forcers modeled in the form of a force by triceps $\left(c_{1} x^{\bullet}\right)$ and a force in biceps $\left(-c_{2} \theta\right)$, where $c_{1}$ and $c_{2}$ are constant and $x^{\bullet}$ is the velocity with which triceps are stretched (or contracted ). We will derive the equation of motion and natural frequency of the forearm of the forearm for small angular dislocation $\theta$. The paper is organized as follows: section 1 describes an introduction about prosthetic research and its control techniques. In Section 2 the mathematical model of a human forearm is described i.e. equation of motion for the angular motion of the forearm about the pivot point is derived, and the motion of the robot arm by a DC motor via a gear is resulting. Section 3 proceed to the study of a finite-dimensional optimal control problem, i.e., a dynamic optimization problem in which the state of the system is linked in time to the application of a control function, by means of the solution to an ordinary differential equation whose right-hand side is shaped by the control. Sections 4 develops a method for constructing state-feedback stabilizing controllers law for a class of dynamical systems where position control algorithms are treated. Section 5 nonlinear state-feedback controller are derived respectively for one-link manipulator model. Lie derivative-based techniques nonlinear controller is simulated in section 5 and Finally, A short conclusion in Section 6 summarizes the study.

\section{MATHMATICAL MODELLING}

Equation of motion for the angular motion of the forearm about the pivot point $\mathrm{O}[9]$ :

$$
I_{0} \theta_{P}^{\bullet \bullet}+m_{2} g b \cos \theta_{P}+m_{1} g \frac{b}{2} \cos \theta_{P}-F_{2} a_{2}+F_{1} a_{1}=0
$$

Where $\theta_{P}$ the angular displacement of the forearm is, $I_{0}$ is the mass of inertia of the forearm and the mass carried:

$I_{0}=m_{2} b^{2}+\frac{1}{3} b^{2} m_{1}$ 
And the forces in the biceps and triceps muscles $\left(F_{2}\right.$ and $\left.F_{1}\right)$ are given by

$F_{2}=-c_{2} \theta_{P}$

$F_{1}=c_{1} x^{\bullet}=c_{1} a_{1} \theta_{P}^{\bullet}$

Where the linear velocity of the triceps can be expressed as

$x^{\bullet} \approx a_{1} \theta_{P}^{\bullet}$

Using Equations (2)-(4), equation (1) can be rewritten as

$$
\begin{aligned}
& I_{0} \theta_{P}^{\bullet \bullet}+\left(m_{2} g b+\frac{1}{2} m_{1} g b\right) \cos \theta_{P}+c_{2} a_{2} \theta_{P} \\
& +c_{1} a_{1}^{2} \theta_{P}^{\bullet}=0
\end{aligned}
$$

Let the forearm undergo small angular displacement $(\theta)$ about the static equilibrium position, $\bar{\theta}$, so that

$\theta_{P}=\bar{\theta}+\theta$

Using Taylor's series expansion of $\cos \theta_{P}$ about $\bar{\theta}_{P}$, the static equilibrium position, can be expressed as (for small value of $\theta$ )

$\cos \theta_{P}=\cos (\bar{\theta}+\theta) \approx \cos \bar{\theta}-\theta \sin \bar{\theta}$

Using $\theta_{P}^{\bullet \bullet}=\theta^{\bullet \bullet}$ and $\theta_{P}^{\bullet}=\theta^{\bullet}$, Equation (6) can be expresses as

$I_{0} \theta_{P}^{\bullet \bullet}+\left(m_{2} g b+\frac{1}{2} m_{1} g b\right)(\cos \bar{\theta}-\sin \bar{\theta} \theta)$

$+c_{2} a_{2}(\bar{\theta}+\theta)+c_{1} a_{1}^{2} \theta^{\bullet}=0$

$I_{0} \theta^{\bullet \bullet}+\left(m_{2} g b+\frac{1}{2} m_{1} g b\right) \cos \bar{\theta}-$

$\sin \bar{\theta}\left(m_{2} g b+\frac{1}{2} m_{1} g b\right) \theta+c_{2} a_{2} \bar{\theta}+c_{2} a_{2} \theta+c_{1} a_{1}^{2} \theta^{\bullet}=0$

Notice that the static equilibrium equation of the forearm at

$\theta_{P}=\bar{\theta}$, Eq.(6) is given by

$\left(m_{2} g b+\frac{1}{2} m_{1} g b\right) \cos \bar{\theta}+c_{2} a_{2} \bar{\theta}=0$

In view of Equation (10), Equation (9) becomes

$\left(m_{2} b^{2}+\frac{1}{3} b^{2} m_{1}\right) \theta^{\bullet \bullet}+c_{1} a_{1}^{2} \theta^{\bullet}$

$+\left\{c_{2} a_{2}-\sin \bar{\theta} g b\left(m_{2}+\frac{1}{2} m_{1}\right)\right\} \theta=0$

which denotes the equation of motion of the forearm. The undamped natural frequency of the forearm can be expressed as:

$$
\omega_{n}=\sqrt{\frac{c_{2} a_{2}-\sin \bar{\theta} g b\left(m_{2}+\frac{1}{2} m_{1}\right)}{b^{2}\left(m_{2}+\frac{1}{3} m_{1}\right)}}
$$

The design of fully functioning artificial arms with physiological speeds-of- response and strength (or better) that can be controlled almost without thought is the goal of upper extremity prosthetics research. Unfortunately, current prosthetic components and interface techniques are still a long way from realizing this goal [1]. By far the most common actuator for electrically powered prostheses is the permanent magnet dc electric motor with some form of transmission. While there is much research into other electrically powered actuator technologies, such as shape memory alloys and electro active polymers, none is to the point where it can compete against the dc electric motor.

In terms of artificial arm, consider a model of one-link robot manipulator shown in Figure 2. Many different types of drive mechanisms have been devised to allow wrist and forearm drive motors and gearboxes to be mounted close to the first and second axis of rotation, thus minimizing the extended mass of the arm. The motion of the robot arm is controlled by a DC motor via a gear [10]. The DC motor is armaturecontrolled and its schematic is shown in Figure 3.The torque delivered by the motor is: $T_{m}=K_{m} i_{a}$, where $k_{m}$ is the motor-torque constant, and $i_{a}$ is the armature current. Let $\mathrm{N}$ denote the gear ratio. Then we have

$\frac{\theta_{P}}{\theta_{m}}=\frac{\text { radius of motor gear }}{\text { radius of arm gear }}=\frac{\text { Number of teeth } \text { motor gear }}{\text { Number of teeth arm gear }}=\frac{1}{N}$

The work done by gears are proportional to their number of teeth and the work done by the gears must be equal. Let $T_{P}$ denote the torque applied to the robot arm. Then,

$T_{P} \theta_{P}=T_{m} \theta_{m}$. Thus, the torque applied the $\operatorname{rod}$ is $T_{P}=N T_{m}=N K_{m} i_{a}$. We use Newton's second law to write the equation modeling the arm dynamics,

$\left(m_{2} b^{2}+\frac{1}{3} b^{2} m_{1}\right) \theta_{P}^{\bullet \bullet}=\left(m_{2} g b+\frac{1}{2} m_{1} g b\right) \cos \theta_{P}+N K_{m} i_{a}$

where $\mathrm{g}=9.8 \mathrm{~m} / \mathrm{sec}^{2}$ is the gravitational constant. Applying Kirchhoff's voltage law to the armature circuit yields

$L_{a} \frac{d i_{a}}{d t}+R_{a} i_{a}+k_{b} N \frac{d \theta_{P}}{d t}=u$

Where $k_{P}$ is the back emf constant. We can now construct a third-order state-space model of the one-link robot. For this we choose the following state variables:

$x_{1}=\theta_{P}, x_{2}=\frac{d \theta_{P}}{d t}=\omega_{P}, x_{3}=i_{a}$,

Then, the model in state-space format is

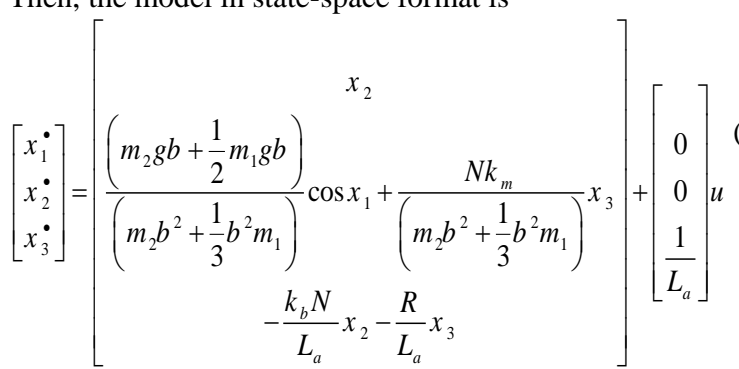

Reasonable parameters of the robot are: $\mathrm{m}_{2}=5 \mathrm{~kg}, \mathrm{~m}_{1}=2 \mathrm{~kg}$, $\mathrm{b}=30 \mathrm{~cm}, \mathrm{~N}=10, \mathrm{k}_{\mathrm{m}}=0.1 \mathrm{Nm} / \mathrm{A}, \mathrm{k}_{\mathrm{b}}=0.1 \mathrm{~V}$ sec/rad, $\mathrm{R}_{\mathrm{a}}=1 \Omega$, $\mathrm{L}_{\mathrm{a}}=100 \mathrm{mH}$. Then the robot model takes the form: 


$$
\left[\begin{array}{c}
x_{1}^{\bullet} \\
x_{2}^{\bullet} \\
x_{3}^{\bullet}
\end{array}\right]=\left[\begin{array}{c}
x_{2} \\
34.59 \cos x_{1}+1.96 x_{3} \\
-10 x_{2}-10 x_{3}
\end{array}\right]+\left[\begin{array}{c}
0 \\
0 \\
10
\end{array}\right] u
$$

We assume that the output $\mathrm{y}$, is

$$
y=x_{1}
$$

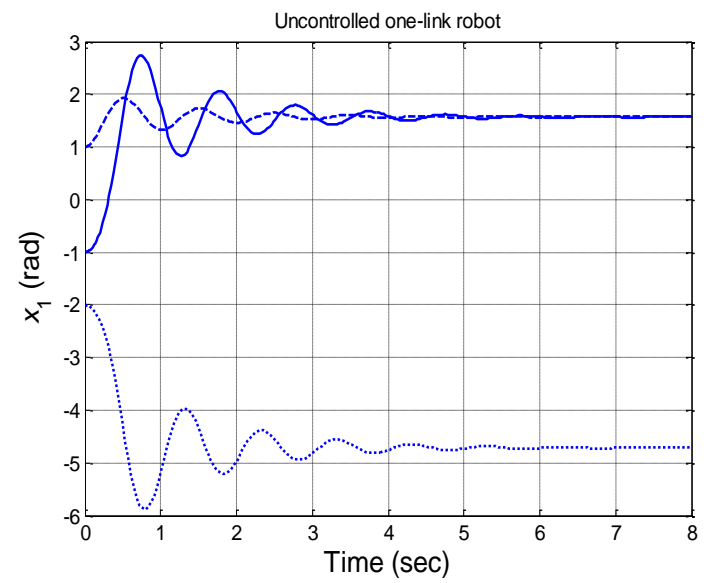

Fig. 4: Plots of the one-link manipulator's, versus time for three different initial angles $\theta(0)$.

Time histories of state trajectories of the uncontrolled nonlinear system of the model by (16) and (17) $x_{1}(t)=\theta(t)$ versus time is shown in Figure 4. The manipulator is driven by $u=0$, initial conditions $x_{1}(0)=1,-1$ and -2 and $x_{2}(0)=0$ and $x_{3}(0)=0$

It is clear that drastic change response due to the initial conditions and also the system has more than one equilibrium points.

\section{FINITE TIME OPTIMAL \\ REGULATOR DEIGN}

The object of the optimal regulator design is to determine the optimal control law $u^{*}(x, t)$ which can transfer the system from its initial state to the final state (with zero system input) such that a given performance index is minimized. The performance index is selected to give the best trade-off between performance and cost of control. The performance index that is widely used in optimal control design is known as the quadratic performance index and is based on minimumerror and minimum-energy criteria [11-14]. Consider the plant described by

$\dot{x}(t)=F(t) x(t)+G(t) u(t), \quad$ given $\mathrm{x}\left(\mathrm{t}_{0}\right)$

We assume that there are no constraints on the control input $u$. with the entries of $F(t), G(t)$ assumed continuous. Let the matrices $\mathrm{Q}(\mathrm{t})$ and $\mathrm{R}(\mathrm{t})$ have continuous entries, be symmetric, and be nonnegative and positive definite, respectively.

The problem is to find the vector $\mathrm{k}(\mathrm{t})$ of the control law

$u(t)=-k(t) x(t)$

which minimizes the value of a quadratic performance index $\mathrm{J}$

of the form $\quad J=\int_{t_{0}}^{t_{f}}\left(x^{\prime} Q x+u^{\prime} R u\right) d t$
Subject to dynamic plant equation in (18). In (20) Q is a positive semidefinite matrix and $\mathrm{R}$ is a real symmetric matrix. $\mathrm{Q}$ is positive semi definite if all its principal minors are nonnegative. The choice of the elements of $\mathrm{Q}$ and $\mathrm{R}$ allows the relative weighting of individual state variables and individual control inputs. To obtain a formal solution, we can use the method of Lagrange multipliers $\lambda$. The constraint problem is solved by augmenting (18) into (20) using an nvector of Lagrange multipliers, $\lambda$. The problem reduces to the minimization of the following unconstrained function

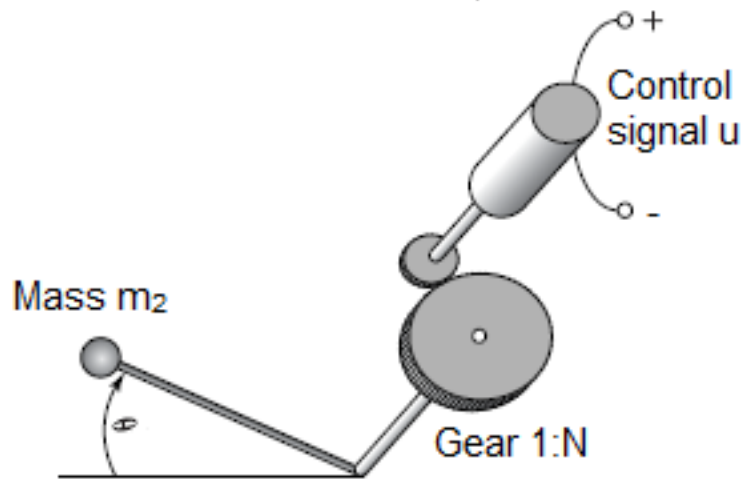

Fig 2: A manipulator of length $b_{1}$ and mass $m_{1}$ controlled by a DC motor via a gear.

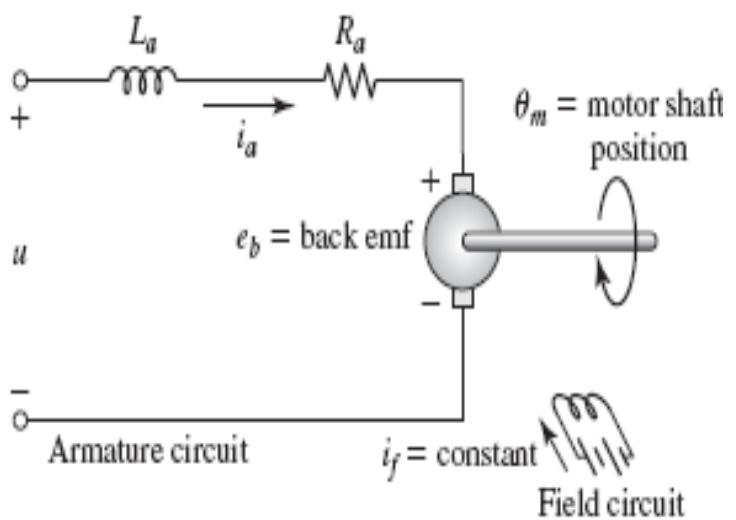

Fig 3: Schematic of an armature controlled DC-Motor

$$
\ell(x, \lambda, u, t)=\left(x^{T} Q x+u^{T} R u\right)+\lambda^{T}(F x+G u-\dot{x})
$$

The optimal values (denoted by the subscript $*$ ) are found by equating the partial derivatives to zero.

$$
\begin{aligned}
& \frac{\partial \ell(x, \lambda, u, t)}{\partial \lambda}=F x^{*}+G u^{*}-\dot{x}^{*}=0 \Rightarrow \dot{x}^{*}=F x^{*}+G u^{*} \\
& \frac{\partial \ell(x, \lambda, u, t)}{\partial u}=2 R u^{*}+\lambda^{T} B=0 \Rightarrow u^{*}=-\frac{1}{2} R^{-1} \lambda^{T} B \\
& \frac{\partial \ell(x, \lambda, u, t)}{\partial x}=2 x^{T *} Q+\dot{\lambda}^{T}+\lambda^{T} F=0 \Rightarrow \\
& \dot{\lambda}=-2 Q x^{*}-F^{T} \lambda
\end{aligned}
$$

Assume that there exists a symmetric, time varying positive definite matrix $\mathrm{p}(\mathrm{t})$ satisfying

$$
\lambda=2 p(t) x^{*}(t)
$$

Substituting (25) into (23) gives the optimal closed-loop control law

$u^{*}(t)=-R^{-1} B^{\prime} p(t) x^{*}(t)$

Obtaining the derivative of (25), we have 
$\dot{\lambda}=2\left(\dot{p}(t) x^{*}(t)+p \dot{x}^{*}\right)$

Finally equating (24) with (27), we obtain

$\dot{p}(t)=-p(t) A-A^{\prime} p(t)-Q+P(t) B R^{-1} B^{\prime} p(t)$

The above equation is referred to as the matrix Riccati differential equation. The boundary condition for (28) is $p\left(t_{f}\right)=0$. Therefore, (28) must be integrated backward in time. Since a numerical solution is performed forward in time, a dummy time variable $\tau=t_{f}-t$ is replaced for time t. Once the solution to (28) is obtained the solution of the state equation (22) in conjunction with the optimum control equation (26) is obtained. $\mathrm{P}(\mathrm{t})$ is the matrix of proportionality between the costate $\dot{p}(t)$ and state $\dot{x}(t)$. This matrix of proportionality is fully specified by the state transition matrix of the Hamiltonian system, Provided the inverse in (28) exists at all times between the initial time and final time.

A matlab function is developed for finite time domain solution of the Riccati differential equation. The function returns the solution of the matrix $P(t)$, the optimal feedback gain vector $K(t)$, and the initial state response. In order to use this function, the user must declare the function containing system matrices and the performance index matrices in an Mfile named.

For linear time-invariant systems, since $\dot{p}=0$, when the process is infinite duration, that is $t_{f}=\infty$, the equation (28) reduces to the algebraic Riccati equation.

$0=p A+A^{\prime} p+Q-P B R^{-1} B^{\prime} p$

Under the controlling of formula (19), the controlled system (18) takes the strong stability and robustness, which can be expressed in the following properties:

\subsection{Stability}

The closed-loop controlled system of (18) and (19) is $\dot{x}=(F-G k) x, K(t)=B^{-1} B^{T} P(t), t \geq 0$, which is asymptotically stable in the large, i.e. its eigenvalues satisfy $R_{e}\left[\lambda_{i}(F-G k)\right]<0, i=1,2, \ldots, n$. In which $\lambda_{i}$ (.) denote $\mathrm{i}^{\text {th }}$ eigenvalue of matrix, $R_{e}$ (.) denotes the real part of a complex.

\subsection{Robustness}

If we let weighting matrixes satisfy the following condition, $R=\operatorname{diag}\left\{r_{1}, r_{2}, \ldots, r_{p}\right\}, r_{i}>0$ then each feedback controlled loop has the performances as follows: At least \pm 60 degrees' phase margin; Gain margin is from $1 / 2$ to $\infty$.

\section{FOREARM LINEARIZATION}

In this forearm model, we linearize the model derived in Section 2. Recall that the equations modeling the system have the form in equation (16) and (17) of an equilibrium state of the form $x_{e}=\left[\begin{array}{lll}x_{1 e} & x_{2 e} & x_{3 e}\end{array}\right]=\left[\begin{array}{lll}\frac{\pi}{2} & 0 & 0\end{array}\right]$

The Jacobian matrix of the model evaluated at the equilibrium state is

$$
\left.\frac{\partial f}{\partial x}\right|_{x=x_{e}}=\left[\begin{array}{ccc}
0 & 1 & 0 \\
-34.59 & 0 & 1.96 \\
0 & -10 & -10
\end{array}\right] \text { and }\left.\frac{\partial f}{\partial u}\right|_{u=u_{e}}=\left[\begin{array}{c}
0 \\
0 \\
10
\end{array}\right]
$$

Hence, the linearized model of the forearm system can be represented as:

$$
\begin{aligned}
& {\left[\begin{array}{l}
\Delta \dot{x}_{1} \\
\Delta \dot{x}_{2} \\
\Delta \dot{x}_{3}
\end{array}\right]=\left[\begin{array}{ccc}
0 & 1 & 0 \\
-34.59 & 0 & 1.96 \\
0 & -10 & -10
\end{array}\right]\left[\begin{array}{l}
\Delta x_{1} \\
\Delta x_{2} \\
\Delta x_{3}
\end{array}\right]+\left[\begin{array}{c}
0 \\
0 \\
10
\end{array}\right] \Delta u, \text { and }} \\
& \Delta y=\left[\begin{array}{lll}
1 & 0 & 0
\end{array}\right]\left[\begin{array}{l}
\Delta x_{1} \\
\Delta x_{2} \\
\Delta x_{3}
\end{array}\right]
\end{aligned}
$$

The problem is to find the optimal control law that minimize the performance index (20), however, the admissible states and control values are unconstrained. The states are initially at $x 1(0)=2, x 2(0)=0$ and $x 3(0)=-2$. For this system we have

$$
F=\left[\begin{array}{ccc}
0 & 1 & 0 \\
-34.59 & 0 & 1.96 \\
0 & -10 & -10
\end{array}\right], G=\left[\begin{array}{c}
0 \\
0 \\
10
\end{array}\right], Q=\left[\begin{array}{lll}
4 & 0 & 0 \\
0 & 3 & 0 \\
0 & 0 & 2
\end{array}\right], R=\frac{1}{2}
$$

The developed algorithm returns the optimal feedback control gains $K(t)$ and the solution of the state equation $x(t)$ in addition to the control law $\mathrm{u}(\mathrm{t})$. First a function called the system parameters and the matrices $\mathrm{Q}$ and $\mathrm{R}$ must be defined in a file. The program calls upon two functions. The function solves the matrix Riccatie differential equation defined by $\mathrm{p}(\mathrm{t})$. The second function solves the state equation in conjunction with the optimum control equation. This function obtains the numerical solution of the time-domain Riccati equation with the boundary condition specified at final time.

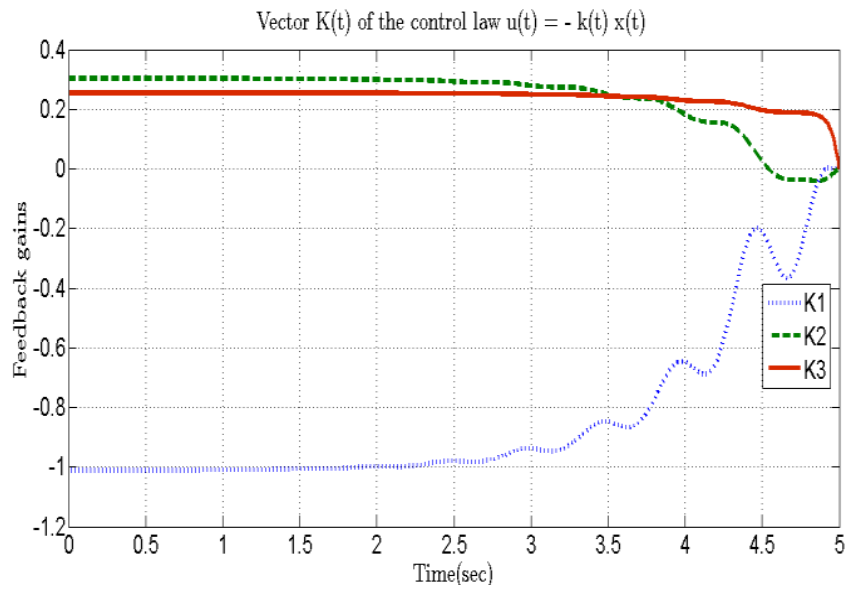

Fig 5: The optimal feedback control gains $K(t)$ versus time

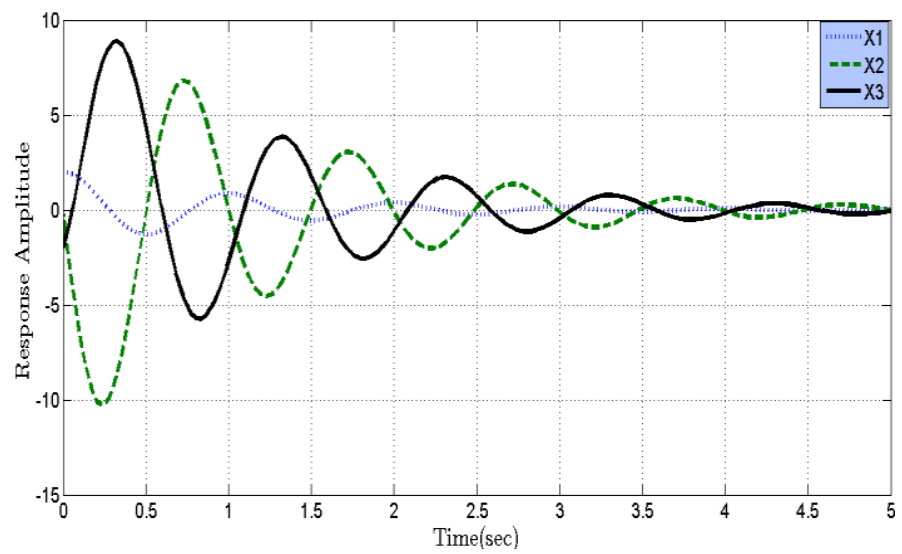


Fig 6: The system response $x(t)$ versus time

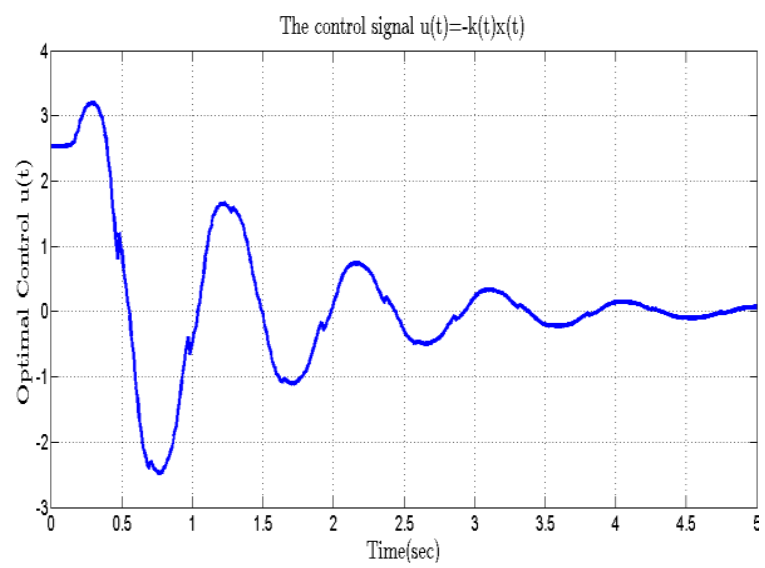

Fig 7: The control law $u(t)$ versus time

$K(t)$ is plotted in Figure 5. Note that the gain exhibits a transient and then approaches a steady -state value far from the final time. In situations where the time interval is long compared to the settling time of this transient, it may be responsible to use only the steady state gain. The feedback system formed using this controller is stable. The closed-loop system is simulated with an initial states of $\mathrm{x} 1(0)=2, \mathrm{x} 2(0)=$ 0 and $\times 3(0)=-2$ and the results are plotted in Figure 6 . Note that states converges to zero, the settling time is roughly 5 seconds, and there is only a small overshoot in the motor shaft angle. The control law $\mathrm{u}(\mathrm{t})$ of this simulation are plotted in Figure 7 and show that zero steady state error is achieved. Looking at the plots, the control input is seen to exceed the normal specifications during the initial transient, but meets that specification after this transient decay to zero. Care must be used in interpreting the meaning of the cost when the test conditions include both initial conditions and inputs.

\section{NONLINEAR CONTROL SYSTEM}

One of the objective of this paper is to devise a method for constructing state-feedback stabilizing control law for a class of dynamical nonlinear systems modeled by

$$
\begin{aligned}
& x^{\bullet}=f(x)+G(x) u, \\
& y=h(x)
\end{aligned}
$$

where $f: R^{n} \rightarrow R^{n}, G: R^{n} \rightarrow R^{n x m}$ and the output map $h: R^{n} \rightarrow R^{p}$ We thus first discuss a method for reducing a nonlinear system model into an equivalent form that is a generalization of the controller form known from linear system theory. In our subsequent discussion, we will be using three types of Lie derivatives $[15,16]$. They are as follows:

1. Derivative of a vector field with respect to a vector field, also known as the Lie bracket.

Given the vector-valued functions $f: R^{n} \rightarrow R^{n}$ and $g: R^{n} \rightarrow R^{n}$, where $f$ and $g$ are $C^{\infty}$ vector fields, their Lie bracket is defined as

$[f, g]=\frac{\partial f}{\partial x} g-\frac{\partial g}{\partial x} f$

2. Derivative of a function with respect to a vector field. Let $h: \mathrm{R}^{n} \rightarrow \mathrm{R}$ be a $C^{\infty} \quad$ function on $R^{n}$. Let
$D h=\nabla h^{T}$, where $\nabla h$ is the gradient (a column vector) of $h$ with respect to $x$. Then, the Lie derivative of the function $\mathrm{h}$ with respect to the vector field $f$, denoted $L_{f} h$ or $L_{f}(h)$, is defined as $L_{f} h=L_{f}(h)=\langle\nabla h, f\rangle=D h f=$ $\frac{\partial h}{\partial x_{1}} f_{1}+\frac{\partial h}{\partial x_{1}} f_{1}+\cdots+\frac{\partial h}{\partial x_{n}} f_{n}$

3. Derivative of $D h$ with respect to the vector field. The Lie derivative of $D h$ with respect to the vector field $f$, denoted $L_{f}(D h)$, is defined as

$L_{f}(D h)=\left(\frac{\partial \nabla h}{\partial x} f\right)^{T}+D h \frac{\partial f}{\partial x}=D L_{f} h=\nabla L_{f}^{T} h$

Our goal now is to construct a $C^{\infty}$ state variable transformation $z=T(x), T(0)=0$

for which there is a $C^{\infty}$ inverse $x=T^{-1}(z)$, such that system model $x^{\bullet}=f(x)+g(x) u$ in the new coordinates has the form

$\left[\begin{array}{c}z_{1}^{\cdot} \\ z_{2}^{\cdot} \\ \vdots \\ z_{n-1}^{\cdot} \\ z_{n}^{\cdot}\end{array}\right]=\left[\begin{array}{c}z_{2} \\ z_{3} \\ \vdots \\ z_{n} \\ \bar{f}_{n}\left(z_{1}, z_{2}, \cdots, z_{n}\right)\end{array}\right]+\left[\begin{array}{c}0 \\ 0 \\ \vdots \\ 0 \\ 1\end{array}\right] u$

$\boldsymbol{x}=\boldsymbol{T}^{-1}(\boldsymbol{z})$ is called a diffeomorphism [13]. The transformation $z=T(x)$ has the for $T=\left[T_{1}, L_{f} T_{1}, \cdots, L_{f}^{n-2} T_{1}, L_{f}^{n-1} T\right]^{T}$

The above means that the problem of constructing the desired transformation $z=T(x)$ is reduced to finding its first component $T_{1}$. The remaining components of $\boldsymbol{T}$ can be successively computed using $T_{1}$. The row vector $\frac{\partial T_{1}(x)}{\partial x}$ is the last row of the inverse of the controllability matrix, provided the controllability matrix is invertible. We denote the last row of the inverse of the controllability matrix by $\boldsymbol{q}(\boldsymbol{x})$. Then, the problem we have to solve is to find $T_{1}=R^{n} \rightarrow R$ such that $\frac{\partial T_{1}(x)}{\partial x}=q(x), T_{1}(0)=0$

where the controllability matrix of the nonlinear system can be expressed in terms of Lie brackets as:

$Q=\left[\begin{array}{llll}\left(a d^{0} f, g\right) & \left(a d^{1} f, g\right) & \cdots & \left(a d^{n-1} f, g\right)\end{array}\right]$

The controllability matrix of the system model in Equations $(16,17)$ is

$Q=\left[\left(a d^{0} f, g\right)\left(a d^{1} f, g\right) \quad\left(a d^{2} f, g\right)\right]=$

$\left[\begin{array}{ccc}0 & 0 & 19.6 \\ 0 & 19.6 & -196 \\ 10 & -100 & 804\end{array}\right]$

The above controllability is of full rank on $\mathrm{R}^{3}$. The last row of its inverse is

$q=\left[\begin{array}{lll}0.051 & 0 & 0\end{array}\right]$, Hence $T_{1}=0.05 x_{1}$ 
Having obtained $T_{1}$, we construct the desired transformation $Z=T(x)$, where

$T(x)=\left[\begin{array}{c}T_{1} \\ L_{f} T_{1} \\ L_{f}\left(L_{f} T\right)\end{array}\right]=\left[\begin{array}{c}0.051 x_{1} \\ 0.051 x_{2} \\ 1.77 \cos x_{1}+0.1 x_{3}\end{array}\right]$

Note that the inverse transformation $x=T^{-1}(z)$ exist and has the form

$T^{-1}(z)=\left[\begin{array}{c}19.6 z_{1} \\ 19.6 z_{2} \\ 10 z_{3}-17.7 \cos \left(19.6 z_{1}\right)\end{array}\right]$

Furthermore $\frac{\partial T}{\partial x}=\left[\begin{array}{ccc}0.05 & 0 & 0 \\ 0 & 0.05 & 0 \\ -1.77 \sin x_{1} & 0 & 0.1\end{array}\right]$

Applying the transformation $Z=T(x)$ to the model of the one-link robot manipulator yields

$z^{\cdot}=\left.\frac{\partial T}{\partial x} f(x)\right|_{x=T^{-1}(z)}+\left.\frac{\partial T}{\partial x} g(x)\right|_{x=T^{-1}(z)} u$

$z^{\cdot}=\left[\begin{array}{c}z_{2} \\ z_{3} \\ -34 z_{2} \sin 19.6 z_{1}-19.6 z_{2}-10 z_{3}+17.7 \cos \left(19.6 z_{1}\right)\end{array}\right]+\left[\begin{array}{l}0 \\ 0 \\ 1\end{array}\right] u$

\section{NONLINEAR STATE FEED-BACK CONTROLLER}

Once the plant model is transformed into the controller form, we can construct a state-feedback controller in the new coordinates and then, using the inverse transformation, represent the controller in the original coordinates [18]. While constructing the controller in the new coordinates, a part of the controller is used to cancel nonlinearities, thus resulting in a linear system in the new coordinates. Then, we proceed to construct the other part of the controller. This part can be designed using linear control methods because the feedback linearized system is linear. The controller form of the one-link manipulator model is given by (43). It is easy to design a stabilizing state-feedback controller in the new coordinates. It takes the form

$$
u=-\bar{f}_{3}\left(z_{1}, z_{2}, z_{3}\right)-\left(k_{1} z_{1}+k_{2} z_{2}+k_{3} z_{3}\right),
$$

where

$\bar{f}_{3}\left(z_{1}, z_{2}, z_{3}\right)=-34 z_{2} \sin 19.6 z_{1}-19.6 z_{2}-10 z_{3}+17.7 \cos \left(19.6 z_{1}\right)$

Suppose that the desired closed-loop poles of the feedback linearized one-link manipulator are to be located at $(-2 \pm j 3.46,8)$ which verify a damping factor $\zeta=0.5$ and natural frequency $\omega_{n}=4 \mathrm{rad} / \mathrm{sec}$. Then, the linear feedback gains $k_{i}=i=1,2,3$ that shift the poles to these desired locations are $k_{1}=128, k_{2}=48 \quad k_{3}=12$. Applying (30) with the above values of the linear feedback gains to the model gives $\dot{z}=\left[\begin{array}{ccc}0 & 1 & 0 \\ 0 & 0 & 1 \\ -128 & -48 & -12\end{array}\right] z$

Next, applying the inverse transformation yields the controller in the original coordinates,

$$
\begin{aligned}
& u=-6.528 x_{1}-1.428 x_{2}-0.2 x_{3}-21.24 \cos x_{1} \\
& -1.81 x_{2} \sin x_{1}
\end{aligned}
$$

In Figure 8, three plots of the manipulator's link angle, $x_{1}=\theta$, versus time are presented. The initial conditions have the form $x(0)=\left[\begin{array}{lll}\theta(0) & 0 & 0\end{array}\right]^{T}$. The control law applied to the manipulator is given by (46).

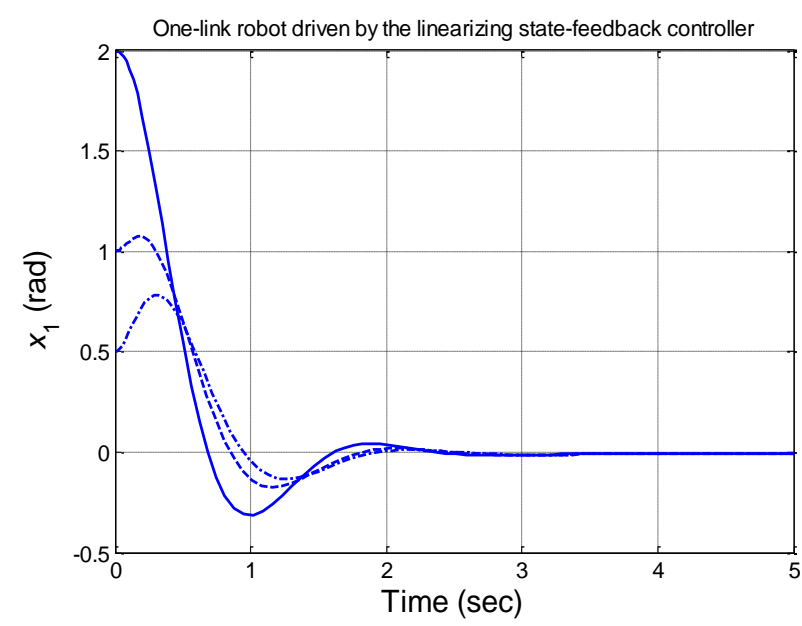

Fig 8: Plots of the one-link manipulator's link angle, $x_{1}=\theta$, versus time for three different initial angles $\theta(0)$. The manipulator is driven by the control law (46).

\section{CONCLUSIONS}

Forearm movement which has the kinematic characteristics was considered as optimal forearm movement. An analysis and design of fully functioning artificial arms with speeds-of response and strength is conducted. The equations of motion and natural frequency of a human forearm are derived. An optimal controller based on Finite Time regulator design that calculates the optimal motor command which drives the arm movement toward the desired state is designed. Synthesis of control law for non-linear systems based on vector fields has been successfully solved. The method is exact and does not require any system linearization. We have managed to design a control law for the non-linear representation of a system, which is controllable and observable, in such a way that the representation of a closed loop system is affine, controllable, observable and asymptotically stable. This fact gives us more flexibility in the choice of the desired behavior of a closed loop system than a linear one. We presented an introduction to qualitative theory of a nonlinear control system, with the main emphasis on controllability and observabilty properties of such systems. We introduced the differential geometric language of vector fields and Lie bracket.. We illustrated our considerations with forearm nonlinear system. The nonlinear controller stabilizes the system and guarantees exponential convergence of the tracking error to zero. Several optimal 
criteria or objective functions, for modeling and control arm movement are demonstrated.

\section{REFERENCES}

[1] Richard F. ff. Weir, 2004 Design of artificial arms and hands for prosthetic applications McGraw-Hill Companies

[2] Weir, R. F. ff., Heckathorne, C. W., and Childress, 2001 D. S. " Cineplasty as a Control Input for Externally Powered Prosthetic Components", Journal of Rehabilitation Research and Development, 38, 4:. 357363, July/August

[3] Yeadon, W. H., and Yeadon, A. W., 2001 Handbook of Small Electric Motors. McGraw-Hill, New York

[4] G. F. Franklin, J. D. Powell, and A. Emami-Naeini, 2006 Feedback Control of Dynamic Systems (5th ed.), Pearson Prentice Hall, Upper Saddle River, NJ

[5] G. J. Bakker and H. Nijmeijer. 1985 Comments on "Control design for multivariable nonlinear time-varying systems." International Journal of Control, 41(6):16271629 ,

[6] N. S. Nise, 2000 Control Systems Engineering (3rd ed.), Wiley, New York

[7] K. Ogata, 2004 System Dynamics (4th ed.), Pearson Prentice Hall, Upper Saddle River, NJ,

[8] Claudio Altafini, May 2001 Geometric Control Methods for Nonlinear Systems and Robotic Applications Doctoral Thesis, Stockholm

[9] Singiresu S. Rao , 2011 Mechanical vibrations,. 5th ed., Prentice Hall Upper Saddle River, NJ

[10] A. Isidori, 1995 Nonlinear Control Systems, third edition. Springer-Verlag, London,

[11] Weiwei Li, 2006 "Optimal Control for Biological Movement Systems" Thesis, University of California, San Diego

[12] Y. Uno, M. Kawato, and R. Suzuki, 1989 "Formation and Control of Optimal Trajectory in Human Multijoint Arm Movement”, Biological Cybernetics 61, 89-101 Springer-Verlag

[13] W. LIy and E. Todorovz , 2007" Iterative linearization methods for approximately optimal control and estimation of non-linear stochastic system International", International Journal of Control, Vol. 80, No. 9, , 14391453

[14] S.I. Biagiola \& J.L. Figueroa, 2011 "Robust model predictive control of Wiener systems" International Journal of Control, Volume 84, Issue 3, , pages 432-444,

[15] R. Marino and P. Tomei., 1995 Nonlinear Control Design: Geometric, Adaptive and Robust., Prentice-Hall Europe, London,
[16] R. Su. July 1982 On the linear equivalents of nonlinear systems. Systems \& Control Letters, 2(1):48-52,

[17] L. R. Hunt, R. Su, and G. Meyer. January 1983 "Global transformations of nonlinear systems." IEEE Transactions on Automatic Control, AC-28(1):24-31,

[18] S. H. ' Zak and C. A. MacCarley. 1986 "State-feedback control of non-linear systems." International Journal of Control, 43(5):1497-1514,

[19] D. Bestle and M. Zeitz. 1983 "Canonical form observer design for non-linear time-variable systems" International Journal of Control, 38(2):419-431,

IJCATM : www.ijcaonline.org ents on Comparative study of on techniques." International Journal or Lontrol, $40(0): 1861-1863$

[21] B. L. Walcott, M. J. Corless, and S. H. Zak. 1987 "Comparative study of non-linear state-observation techniques", International Journal of Control, Vol. 45, No. 6, 2109-2132,

[22] , D. Bestle and M. Zeitz., 1983 "Canonical form observer design for non-linear time-variable systems" International Journal of Control, 38(2):419-431,

[23] A.Mesbaha,, Adrie E.M. Huesmana, Herman J.M. Kramerb, Paul M.J. Van den Hofa, 2011 "A comparison of nonlinear observers for output feedback model-based control of seeded batch crystallization processes", Journal of Process Control 21, 652-666,

[24] A. Ebrahim, G. Murphy, 2008 "Adaptive backstepping control of an induction motor under time-varying load torque and rotor resistance uncertainty", Int. J. of Automation and Control, 2,4: 401 - 417

[25] Frank A. Pintara \& Narayan Yoganandana, 2002 "Dynamic Bending Tolerance of the Human Forearm", Traffic Injury Prevention, 3( 1): 43-48

\section{AUTHOR'S PROFILE}

Gamal A. Elnashar was born in Dakhliah-Egypt on February 17, 1965. In 1988 he received his B.Sc. degree from the department of electrical engineering. His MSc. Degree in the field of automatic control from Military Technical Collage (MTC)-Egypt in 1994

He received his $\mathrm{Ph}$. D. degree from the department of electrical and computer engineering from the Catholic University of America- Washington D. C. in 2000. He has been serving on the MTC research faculty since the year 2000 in the areas of identification, design, and control engineering systems. He worked as a visiting scholar in Virginia Tech.Blacksburg in 2008. He has authored several reports and papers on data acquisition systems, sensors and automatic control-related short courses at MTC. 